\title{
Actin bundling by dynamin 2 and cortactin is implicated in cell migration by stabilizing filopodia in human non-small cell lung carcinoma cells
}

\author{
HIROSHI YAMADA ${ }^{1,3}$, TETSUYA TAKEDA ${ }^{1,3}$, HIROYUKI MICHIUE ${ }^{2}$, \\ TADASHI ABE ${ }^{1,3}$ and $\mathrm{KOHJI}_{\text {TAKEI }}{ }^{1,3}$ \\ Departments of ${ }^{1}$ Neuroscience and ${ }^{2}$ Physiology, Graduate School of Medicine, \\ Dentistry and Pharmaceutical Sciences, Okayama University; ${ }^{3}$ CREST, \\ Japan Science and Technology Agency, Kita-ku, Okayama 700-8558, Japan
}

Received April 6, 2016; Accepted May 25, 2016

DOI: 10.3892/ijo.2016.3592

\begin{abstract}
The endocytic protein dynamin participates in the formation of actin-based membrane protrusions such as podosomes, pseudopodia, and invadopodia, which facilitate cancer cell migration, invasion, and metastasis. However, the role of dynamin in the formation of actin-based membrane protrusions at the leading edge of cancer cells is unclear. In this study, we demonstrate that the ubiquitously expressed dynamin 2 isoform facilitates cell migration by stabilizing F-actin bundles in filopodia of the lung cancer cell line H1299. Pharmacological inhibition of dynamin 2 decreased cell migration and filopodial formation. Furthermore, dynamin 2 and cortactin mostly colocalized along F-actin bundles in filopodia of serum-stimulated H1299 cells by immunofluorescent and immunoelectron microscopy. Knockdown of dynamin 2 or cortactin inhibited the formation of filopodia in serum-stimulated H1299 cells, concomitant with a loss of F-actin bundles. Expression of wild-type cortactin rescued the punctate-like localization of dynamin 2 and filopodial formation. The incubation of dynamin 2 and cortactin with F-actin induced the formation of long and thick actin bundles, with these proteins colocalizing at F-actin bundles. A depolymerization assay revealed that dynamin 2 and cortactin increased the stability of F-actin bundles. These results indicate that dynamin 2 and cortactin participate in cell migration by stabilizing F-actin bundles in filopodia. Taken together, these findings suggest that dynamin might be a possible molecular target for anticancer therapy.
\end{abstract}

Correspondence to: Dr Hiroshi Yamada, Department of Neuroscience, Graduate School of Medicine, Dentistry and Pharmaceutical Sciences, Okayama University, 2-5-1 Shikata-cho, Kita-ku, Okayama 700-8558, Japan

E-mail: hiroyama@md.okayama-u.ac.jp

Key words: actin, cortactin, dynamin, filopodia, migration

\section{Introduction}

Cancer cell migration, invasion, and metastasis are preceded by the formation of pseudopodia such as lamellipodia and filopodia. During these cellular processes, F-actin filaments remodel into a higher order structure and then assemble an intricate cytoskeletal network within cells (1). These dynamic three-dimensional changes are mediated by several actin-bundling and crosslinking proteins, and are essential for supporting filopodia at the leading edge of migrating cells (2).

Dynamin plays an essential role in endocytosis, participating in the membrane fission process (3-5). Dynamin also functions in the formation of actin-rich structures, including lamellipodia and dorsal membrane ruffles $(6,7)$, invadopodia (8), podosomes (9), growth cones (10-12), and phagocytic cups $(13,14)$. Three dynamin isoforms exist, namely, dynamin 1,2 , and 3 (5). Dynamins are characterized by a GTPase domain at the $\mathrm{N}$-terminus, a bundle signaling element, a stalk domain, a phosphoinositide-binding pleckstrin homology domain, and a proline and arginine-rich domain at the C-terminus (PRD) $(15,16)$. The PRD interacts with different proteins that contain the Src-homology-3 (SH3) domain. Of these GTPases, dynamin 2 is ubiquitously expressed.

Cortactin, an F-actin-binding protein, was first identified as an Src substrate (17). Cortactin also participates in cancer cell migration, invasion, and metastasis by regulating actin dynamics at the leading edge of migrating cells (18). Cortactin is composed of an $\mathrm{N}$-terminal acidic domain and a six-and-ahalf tandem repeats domain, which directly binds to F-actin. Cortactin also contains an $\alpha$-helix, a proline-rich region, and an SH3 domain at the C-terminus, which interacts with the PRD of several binding partners (19).

Both dynamin and cortactin are implicated in the dynamics of cancer cells, including migration, invasion, and metastasis (18). In addition, the pharmacological inhibition of dynamin by GTPase inhibitors suppresses specific cellular processes such as the lamellipodial formation and invasion of human osteocarcinoma cells (20) and the growth of human prostate adenocarcinoma cells (21). 
A previous study reported that dynamin 2 binds to cortactin $(7,12)$. A disruption of this protein complex can affect the shape of cancer cells (7), organization of the F-actin network within these cells (22), and structure of growth cones $(11,12)$. However, the role of the dynamin 2-cortactin complex in the dynamics of the actin cytoskeleton in cancer cells is unclear. In this study, we investigated whether dynamin 2 and cortactin regulate the $\mathrm{F}$-actin bundle formation in filopodia in the human non-small cell lung carcinoma cell line H1299.

\section{Materials and methods}

Antibodies and reagents. Rabbit polyclonal anti-dynamin 1 (cat. no. PA1-660; Thermo Fisher Scientific, Waltham, MA, USA) and anti-c-myc (cat. no. C3956; Sigma-Aldrich, St. Louis, MO, USA) antibodies, and a goat polyclonal anti-dynamin 2 (cat. no. sc-6400; Santa Cruz Biotechnology, Santa Cruz, CA, USA) antibody, were purchased. In addition, mouse monoclonal anti- $\beta$-actin (cat. no. A5441, Sigma-Aldrich), Dynasore (cat. no. D7693, Sigma-Aldrich), anti-c-myc (cat. no. sc-40; Santa Cruz Biotechnology), anti-green fluorescent protein (GFP; cat. no. sc-9996, Santa Cruz Biotechnology), and anti-cortactin (cat. no. 05-180; EMD Millipore, Darmstadt, Germany) antibodies were purchased. MitMAB and Dynole 34-2 were purchased from Abcam Biochemicals (Bristol, UK). Alexa Fluor 488-conjugated anti-rabbit IgG, rhodamine-conjugated anti-mouse IgG, and rhodamine or Alexa Fluor 488-labeled phalloidin were obtained from Thermo Fisher Scientific. Purified rabbit skeletal $\alpha$-actinin was purchased from Cytoskeleton, Inc. (Denver, CO, USA). Goat anti-mouse IgG- and goat anti-rabbit IgG-conjugated gold particles were purchased from British BioCell International (Cardiff, UK).

Cell culture. The human non-small cell lung carcinoma cell line H1299 (Cat. no. ATCC CRL-5803; American Type Culture Collection, Manassas, VA, USA) was cultured in Dulbecco's modified Eagle's medium (DMEM, Thermo Fisher Scientific) supplemented with $10 \%$ fetal bovine serum (FBS) at $37^{\circ} \mathrm{C}$ in an atmosphere of $5 \% \mathrm{CO}_{2}$.

Expression and purification of dynamin 2 and cortactin wild-types and mutants. GFP-tagged dynamin 2 cloned into pEGFP-N1 was a kind gift from Dr Mark McNiven (Mayo Clinic, Rochester, MN, USA) (6). His-tagged dynamin 2 produced with the Bac-to-Bac baculovirus expression system (Thermo Fisher Scientific) was a kind gift from Dr Hiroshi Handa (Tokyo Institute of Technology, Tokyo, Japan) (23). The dynamin solution was concentrated using a Centriplus YM50 (Thermo Fisher Scientific) and stored at $-80^{\circ} \mathrm{C}$. The protein suspension (2-5 $\mathrm{mg} / \mathrm{ml}$ protein) was thawed at $37^{\circ} \mathrm{C}$ before use.

The cDNAs encoding full-length rat cortactin and its mutants were prepared by polymerase chain reaction amplification using specific primers (12). Full-length cortactin or 1-450aa (Cort $\triangle \mathrm{SH} 3$ ) was subcloned into the plasmid pGEX-6p vector as BamHI-EcoRI fragments. GST-tagged cortactin W525K was generated by mutating pGEX-6p-cortactin with the QuickChange site-directed mutagenesis kit (Agilent Technologies, Santa Clara, CA, USA). For expression in cells, full-length cortactin or Cort $\triangle \mathrm{SH} 3$ was subcloned into the pEF1 myc-His vector (Thermo Fisher Scientific) as EcoRI-XbaI fragments. The nucleotide sequences of the constructs were verified by DNA sequence analysis. The resulting plasmid was transformed into the bacterial BL21 (DE3) pLysS strain for protein expression. The expression of GST-fusion proteins was induced by $0.1 \mathrm{mM}$ isopropyl-1-thio-D-galactopyranoside at $37^{\circ} \mathrm{C}$ for 3-6 h in LB medium supplemented with $100 \mu \mathrm{g} / \mathrm{ml}$ ampicillin to $\mathrm{A}_{600}=0.8$. The purification of GST-fusion proteins was performed as previously described (24), and the cleavage of the GST with PreScission protease was performed according to the manufacturer's instructions. The protein was purified on a MonoQ column equilibrated in $20 \mathrm{mM}$ Tris- $\mathrm{HCl}$ and $0.2 \mathrm{M}$ $\mathrm{NaCl}, \mathrm{pH}$ 7.7. The eluted protein fraction $(1 \mathrm{mg} / \mathrm{ml}$ protein) was stored at $-80^{\circ} \mathrm{C}$. For the pull-down assay, the proteins were used without cleaving GST.

siRNA-mediated interference. Pre-annealed siRNAs for human dynamin 2 and cortactin, and the negative control siRNA, were synthesized and purified (Thermo Fisher Scientific). The sequences for the siRNAs for human dynamin 2 were as follows: 5'-GGAUAUUGAGGGCAAGAAGtt-3' (sense), 5'-CUUCUUGCCCUCAAUAUCCtt-3' (antisense) for oligo 1; 5'-GCGAAUCGUCACCACUUACtt-3' (sense), 5'-GUAAGUG GUGACGAUUCGCtc-3' (antisense) for oligo 2; and 5'-GGAC UUACGACGGGAGAUCtt-3' (sense), 5'-GAUCUCCCGU CGUAAGUCCtt-3' (antisense) for oligo 3. The sequences for the siRNAs for human cortactin were as follows: CCGAAUG GAUAAGUCAGUCtt-3' (sense), 5'-AGCUGACUUAUCCAU UCGGtc-3' (antisense) for oligo 1; GGUUUCGGCGGCA AAUACGtt-3' (sense), CGUAUUUGCCGCCGAAACCtt-3' (antisense) for oligo 2; and CGAAUAUCAGUCGAAACUUtt-3' (sense), AAGUUUCGACUGAUAUUCGtg-3' (antisense) for oligo 3.

A scrambled siRNA with no significant sequence homology to all mouse, rat, or human gene sequences was used as the negative control. The day before transfection, the cells were plated in 6 -well plates $\left(5 \times 10^{4}\right.$ cells/well). One hundred picomoles of the duplex siRNAs was transfected into the cells using $4 \mu \mathrm{l}$ of Lipofectamine 2000 (Thermo Fisher Scientific). After $72 \mathrm{~h}$, the cells were treated differently according to experimental design. In pilot experiments, we confirmed that all three transfections of siRNA for dynamin 2 and cortactin were effective.

Filopodial formation. H1299 cells were serum-starved for $16 \mathrm{~h}$. Thereafter, the cells were transfected with dynamin 2 siRNAs, cortactin siRNAs, or the control siRNA, followed by incubation with DMEM supplemented with $10 \%$ FBS for $45 \mathrm{~min}$. For the rescue experiments, cortactin was silenced in H1299 cells with oligo 3, and the cells were cultured for $24 \mathrm{~h}$. The cells (1x10\% coverslip) were then transfected with rat wild-type cortactin or cortactin W525K $(0.25 \mu \mathrm{g}$ each) cloned into the pIRES2-AcGFP1 expression vector (Clontech Laboratories, Santa Clara, CA, USA). Thereafter, the cells were stimulated with serum for $45 \mathrm{~min}$, fixed, and stained with Alexa Fluor 488 or rhodamine-labeled phalloidin for visualization of filopodia.

Wound healing assay. H1299 cells were cultured to confluence on glass-bottom dishes (35 mm diameter; AGC Techno Glass Co.Ltd., Tokyo, Japan) in DMEM supplemented with $0.2 \%$ FBS 
for $8 \mathrm{~h}$. Thereafter, the cell layer was wounded with a plastic pipette tip as previously described (25). The cells were washed with DMEM supplemented with $0.2 \%$ FBS and incubated for $8 \mathrm{~h}$ in the presence of Dynasore, Dynole 34-2 or MitMAB at the indicated concentrations. For the negative control, cells were incubated with $1 \%$ dimethyl sulfoxide (DMSO). The cells were visualized by Giemsa staining, followed by the acquisition of phase contrast images from $\geq 20$ randomly selected areas per dish. Areas filled with migrating cells were analyzed with ImageJ software (National Institutes of Health, Bethesda, MD, USA).

Formation of in vitro $F$-actin bundles. For the fluorescent detection of F-actin, non-muscle actin was polymerized in F-buffer (10 mM Tris-HCl, $0.2 \mathrm{mM} \mathrm{DTT,} 0.2 \mathrm{mM} \mathrm{CaCl}_{2}$, $2 \mathrm{mM} \mathrm{MgCl} 2,50 \mathrm{mM} \mathrm{KCl}$, and $0.5 \mathrm{mM} \mathrm{ATP,} \mathrm{pH}$ 7.5) for $1 \mathrm{~h}$. Thereafter, $3.3 \mu \mathrm{M}$ F-actin was incubated with $5 \mu \mathrm{M}$ dynamin 1 or 2 and cortactin for $1 \mathrm{~h}$, followed by an additional 30 min with $3 \mu \mathrm{M}$ Alexa Fluor 488-phalloidin. The samples were spread onto glass slides and mounted, and the F-actin bundles were observed under an epifluorescent microscope.

For the immunolocalization of dynamin and cortactin, F-actin bundles were incubated with dynamin 1 or 2 and cortactin for $30 \mathrm{~min}$ with $3 \mu \mathrm{M}$ phalloidin to stabilize the filaments, followed by centrifugation at 5,000 x g for $10 \mathrm{~min}$. The pellet was resuspended with $50 \mu 1$ of F-buffer and then immunostained in suspension for 30 min with $1 \mu \mathrm{l}$ of primary antibody. The mixture was centrifuged at 5,000 x g, and the pellet was washed with F-buffer. The samples were incubated with secondary antibodies and washed as previously done for the primary antibody. All steps were performed at room temperature. The samples were spread onto glass slides and mounted. The samples were examined under a spinning disc confocal microscope system (CSU10, Yokogawa Electric Co., Tokyo, Japan) combined with an inverted microscope (IX-71, Olympus Optical Co., Ltd., Tokyo, Japan) and a CoolSNAP-HQ camera (Roper Technologies, Sarasota, FL, USA). The confocal system was controlled by MetaMorph software (Molecular Devices, Sunnyvale, CA, USA). Images were processed using Adobe Photoshop CS3 or Illustrator CS3 software.

Immunoprecipitation assay. For the immunoprecipitation assay, H1299 cells were co-transfected with GFP-tagged dynamin 2 and either myc-tagged cortactin or cortactin $\triangle \mathrm{SH} 3$. The cells were lysed with $1 \% \mathrm{NP}-40,100 \mathrm{mM} \mathrm{KCl}, 0.5 \mathrm{mM}$ EDTA, $10 \mathrm{mM} \mathrm{NaF}$, and $20 \mathrm{mM}$ HEPES/KOH, pH 7.4, and a protease inhibitor cocktail tablet (Roche Diagnostics, Basel, Switzerland). The protein complexes were immunoprecipitated from $1 \mathrm{mg}$ of cell extract using either $5 \mu \mathrm{g}$ of the polyclonal anti-myc antibodies or preimmune $\mathrm{IgG}$, and then visualized by western blotting with a monoclonal anti-GFP or anti-myc antibody.

Immunostaining and fluorescent microscopy. H1299 cells were fixed with $4 \%$ paraformaldehyde and stained by immunofluorescence as previously described (12).

Transmission electron microscopy. Specimens were embedded for immunoelectron microscopy as previously described (12). In brief, H1299 cells were fixed with cytoskeleton buffer $(10 \mathrm{mM}$ 2-(N-morpholino)ethanesulfonic acid, $150 \mathrm{mM} \mathrm{NaCl}, 5 \mathrm{mM}$ EGTA, $5 \mathrm{mM} \mathrm{MgCl}_{2}$, and $5 \mathrm{mM}$ glucose, $\mathrm{pH}$ 6.0) containing $10 \mu \mathrm{g} / \mathrm{ml}$ phalloidin, $0.1 \%$ Triton X-100, and $3 \%$ formaldehyde for $1 \mathrm{~min}$. The cells were then fixed for an additional $30 \mathrm{~min}$ without Triton X-100, followed by washing with $5 \mu \mathrm{g} / \mathrm{ml}$ phalloidin in phosphate-buffered saline (PBS). After incubation in blocking solution $(10 \mu \mathrm{g} / \mathrm{ml}$ phalloidin, $2 \mathrm{mg} / \mathrm{ml} \mathrm{BSA}$, and $100 \mathrm{mM}$ glycine in PBS), the specimens were incubated with a primary antibody diluted in blocking solution, washed with $5 \mu \mathrm{g} / \mathrm{ml}$ phalloidin in PBS, incubated with goat anti-mouse or rabbit anti-goat IgG conjugated to $10-\mathrm{nm}$ gold particles, and then fixed with $2.5 \%$ glutaraldehyde and $5 \mu \mathrm{g} / \mathrm{ml}$ phalloidin in PBS. The specimens were post-fixed with $1 \% \mathrm{OsO}_{4}$ in $0.1 \mathrm{M}$ sodium cacodylate buffer for $1 \mathrm{~h}$, dehydrated, and embedded in EPON 812 for ultrathin sectioning. Cross-sections were visualized under a Hitachi $\mathrm{H}-7100$ transmission electron microscope.

Determination of filopodial length. For the measurement of the filopodial length, H1299 cells were fixed and stained with rhodamine- or Alexa Fluor 488-conjugated phalloidin. Membrane protrusions supported with F-actin bundles were defined as filopodia, and digital images were acquired at 400-1,000x magnifications. Up to five filopodia for each cell were randomly selected, and their lengths were measured with ImageJ software.

Statistical analysis. Data were analyzed for statistical significance using KaleidaGraph software (version 4.1) for the Macintosh (Synergy Software Inc., Essex Junction, VT, USA). Analysis of variance and Tukey's honest significant difference post hoc test were applied for more than two different groups, and Student's t-test was applied for two different groups. P-values of $<0.05$ and 0.001 were considered as statistically significant.

\section{Results}

Inhibition of dynamin decreases the migration of the human non-small cell lung carcinoma cell line H1299. To determine whether dynamin 2 is involved in cell migration, the effects of dynamin inhibition on cell migration were determined by a wound healing assay. Cell migration decreased after treatment of cells with Dynasore (26), Dynole 34-2 (27), and MitMAB (28) (Fig. 1A). Dynasore ( $80 \mu \mathrm{M})$ inhibited cell migration by $\sim 80 \%$ compared to that of control cells, whereas Dynole 34-2 and MitMAB inhibited cell migration by $20-40 \%$ (Fig. 1B). These results indicate that dynamin is important for the migration of H1299 cells.

Dynamin 2 colocalizes with cortactin along $F$-actin bundles in filopodia of H1299 cells. Fig. 1 shows that dynamin is involved in cell migration mediated by pseudopodia. Thus, we investigated whether dynamin 2 participates in filopodial formation in $\mathrm{H} 1299$ cells. Because cortactin functions with dynamin 1 in the bundling of F-actin, which is important for the stability of filopodia in human neuroblastoma cell line SH-SY5Y (12), dynamin 2 and cortactin were immunostained in serum-stimulated H1299 cells. H1299 cells formed numerous filopodia 

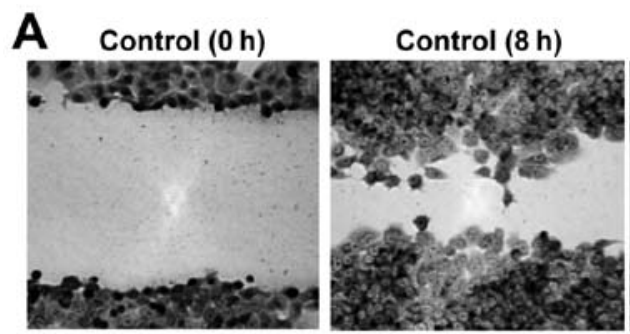
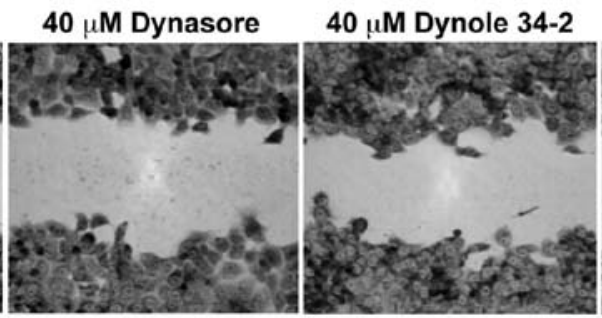

\section{$10 \mu \mathrm{M}$ MitMAB}

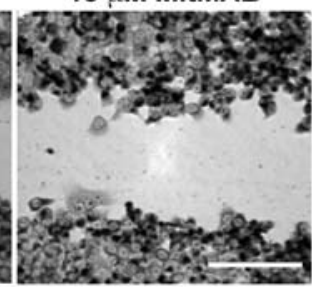

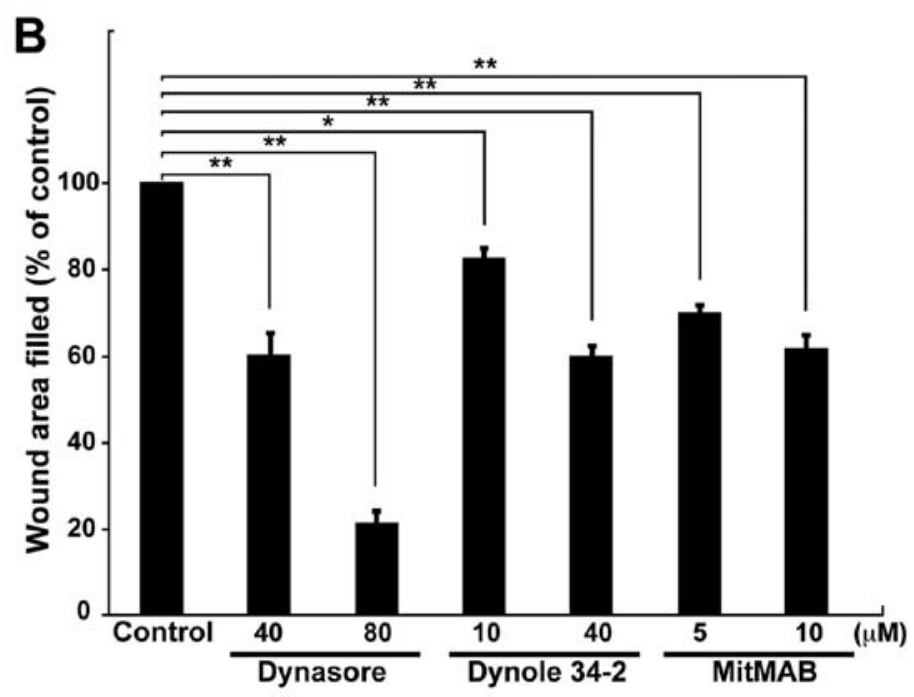

Figure 1. Dynamin GTPase inhibitors inhibit the migration of H1299 cells. (A) Representative images acquired by light microscopy showing cell migration in a wound healing assay. Confluent H1299 cells were wounded and then incubated for $8 \mathrm{~h}$ in the presence or absence of dynamin GTPase inhibitors at the indicated concentrations. For the negative control (control $0 \mathrm{~h}$ ), cells were incubated with 1\% DMSO. Scale bar, $200 \mu \mathrm{m}$. (B) Morphometric analysis of the wound area filled by migrating cells after treatment with inhibitors at the indicated concentrations. The changes were normalized to the control. Results represent the means \pm SEM of three independent experiments.

after serum stimulation (Fig. 2A). Furthermore, dynamin 2 and cortactin colocalized to F-actin bundles in filopodia as bright dots (Fig. 2A). The negative controls showed little immunoreactivity for dynamin 2 and cortactin (Fig. 2B). In addition, immunoelectron microscopy revealed that both proteins localized to F-actin bundles in filopodia (Fig. 2C).

These results prompted us to examine the possible interaction of dynamin 2 and cortactin by immunoprecipitation. Exogenously expressed dynamin 2-GFP was co-precipitated with full-length cortactin-myc using a polyclonal anti-myc antibodies and H1299 cell lysates (Fig. 2D, left). Cort $\Delta \mathrm{SH} 3$-myc, a dynamin 2 binding deficient mutant that lacks its SH3 domain, was unable to precipitate dynamin 2 (Fig. 2D, right). Taken together, these results illustrate that these proteins interact at F-actin bundles in filopodia of H1299 cells.

Dynamin 2 and cortactin are required for serum-induced filopodial formation in H1299 cells. To examine the role of dynamin 2 in filopodial formation, dynamin 2 was silenced in H1299 cells by RNAi. Compared with the control, knockdown of dynamin 2 in $\mathrm{H} 1299$ cells with specific siRNAs reduced its level by $\sim 95 \%$ as revealed by western blotting (Fig. 3A). Compared with the length of filopodia in serum-stimulated control cells $(10.2 \pm 0.5 \mu \mathrm{m})$, dynamin 2 knockdown decreased filopodial extension in silenced cells $(4.7 \pm 0.6 \mu \mathrm{m}$ ) (Fig. 3B and $\mathrm{C}$ ). In addition, dynasore inhibited filopodial extension $(2.4 \pm 0.08 \mu \mathrm{m})$. This effect was rescued after the inhibitor was removed $(8.1 \pm 2.4 \mu \mathrm{m})$ (Fig. 3D and E).
We also examined the effects of cortactin knockdown by RNAi on filopodial formation. Compared with the control, knockdown of cortactin reduced its level by $~ 95 \%$ as revealed by western blotting (Fig. 4A). Compared with the length of filopodia in control cells $(10.2 \pm 0.39 \mu \mathrm{m})$, cortactin knockdown also decreased filopodial extension after serum-stimulation $(5.6 \pm 0.17 \mu \mathrm{m})$ (Fig. 4B and C). The inhibition of filopodial formation in cortactin-silenced cells was rescued by exogenous expression of wild-type cortactin $(10.8 \pm 0.54 \mu \mathrm{m})$ but not by cortactin W525K, a binding-defective mutant of dynamin 2 (29) (Fig. 4D and E). In addition, the punctate-like localization of dynamin 2 along F-actin bundles reappeared in wild-type cortactin expressing cells (Fig. 4F, right). These results indicate that dynamin 2 and cortactin are required for filopodial formation.

F-actin bundling by the dynamin 2-cortactin complex stabilizes F-actin. The effects of dynamin 2 and cortactin on the formation of F-actin bundles were examined in vitro. In this experiment, preformed F-actin were incubated with or without cortactin and dynamin 2 in the presence of GTP. F-actin alone appeared as uniform filaments (Fig. 5A, actin alone). The addition of dynamin 2 to F-actin filaments did not cause any visible change in their distribution (Fig. 5A, + Dyn2). However, F-actin incubated with wild-type cortactin, cortactin W525K or cortactin $\triangle \mathrm{SH} 3$ often formed small clusters (Fig. $5 \mathrm{~A},+$ Cort $\mathrm{WT},+$ Cort W525K, or + Cort $\Delta \mathrm{SH} 3$ ), consistent with a previously published report (12). The presence of both dynamin 2 
A

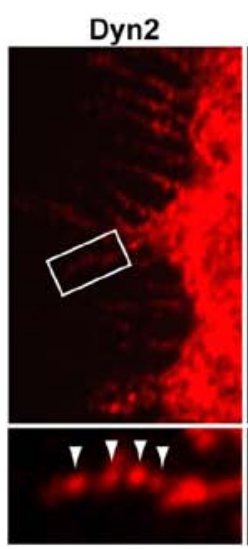

C

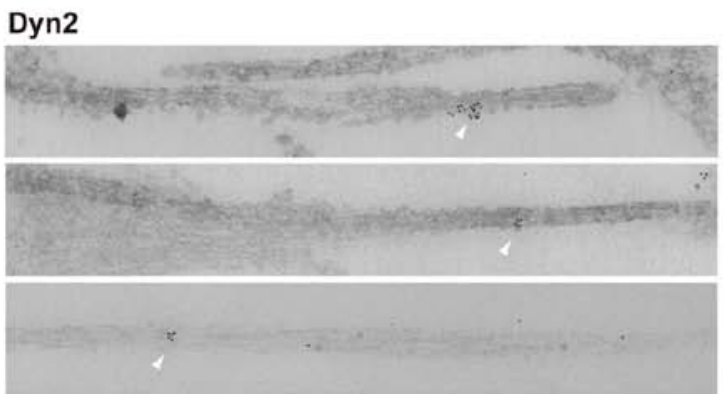

Cort
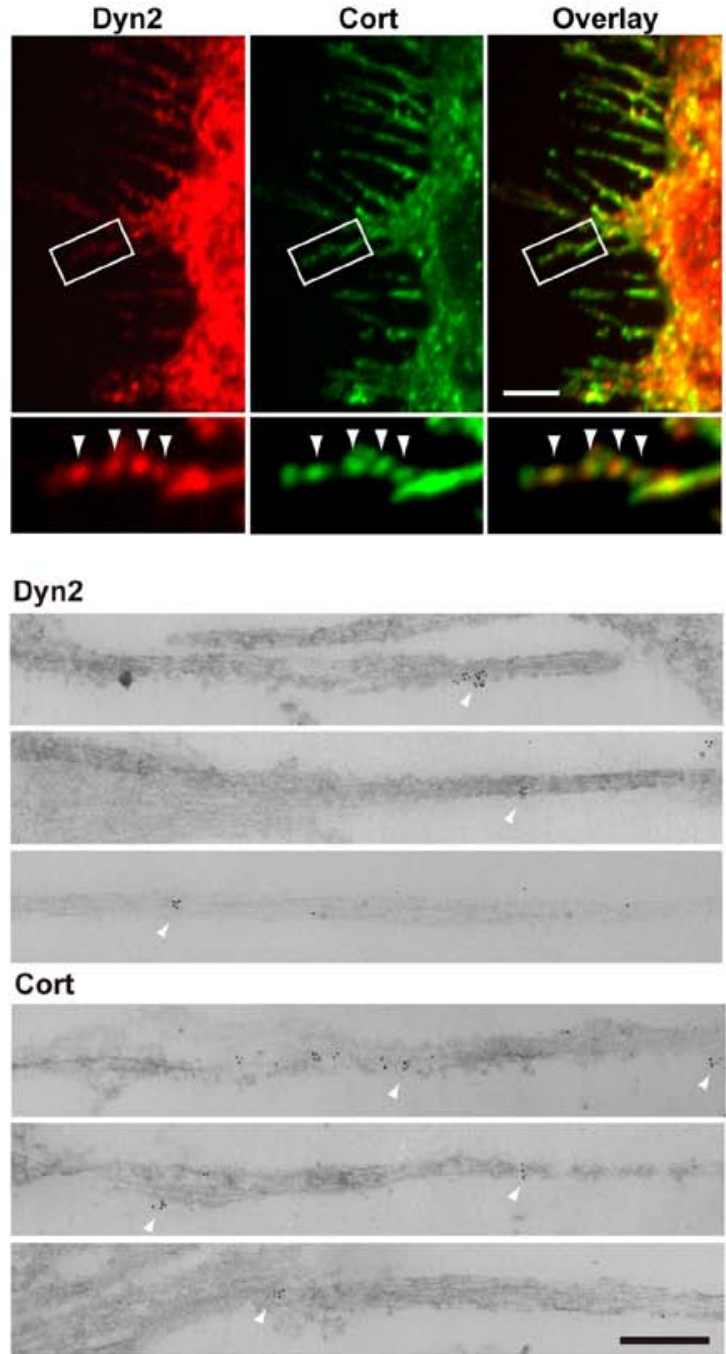

B

\section{D}
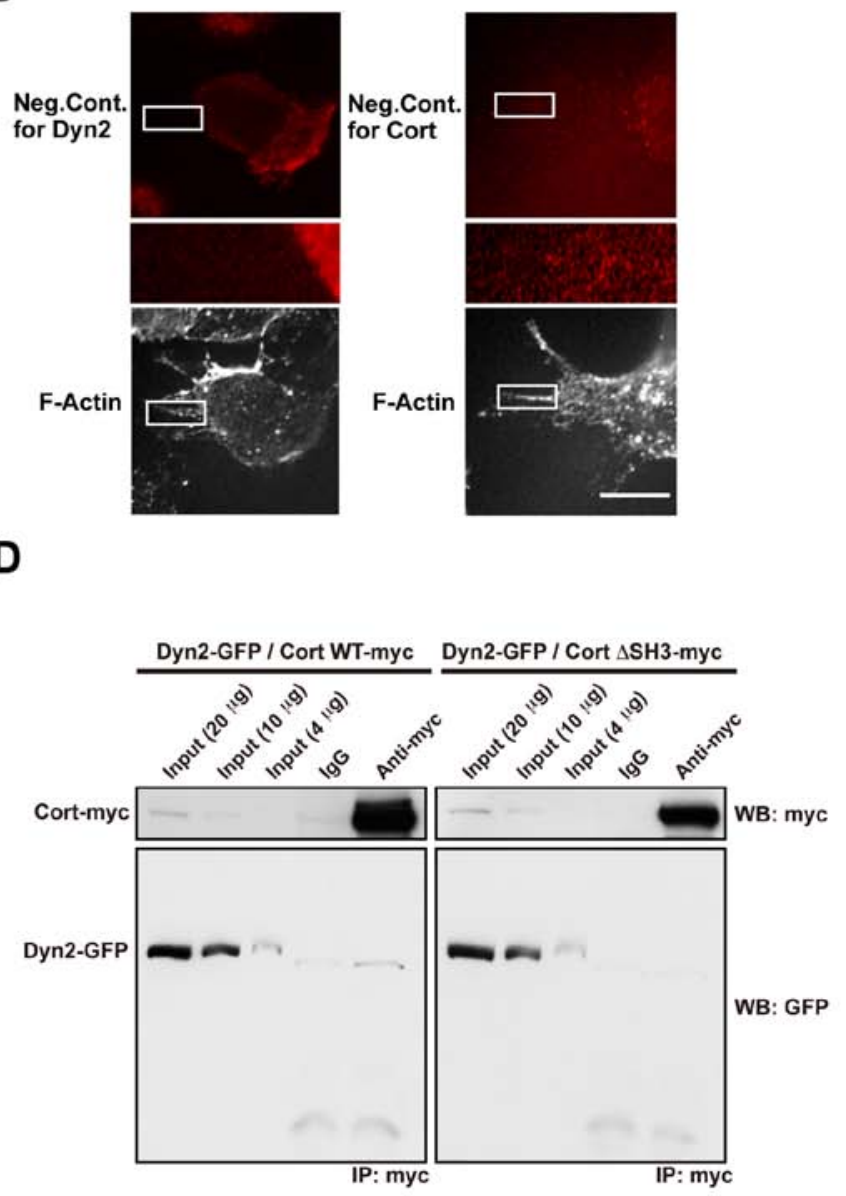

Figure 2. Dynamin 2 colocalizes with cortactin along F-actin bundles in filopodia of serum-stimulated H1299 cells. (A) Colocalization of dynamin 2 (Dyn2, left) and cortactin (Cort, middle) by double-immunofluorescent staining in filopodia of serum-stimulated H1299 cells. Boxed areas correspond to enlarged images shown below. Dynamin 2- and cortactin-positive puncta were present periodically along F-actin bundles in filopodia (arrowheads). Scale bar, $5 \mu \mathrm{m}$ (upper panels), $1.6 \mu \mathrm{m}$ (lower panels). (B) In the negative controls, the primary antibodies were omitted for dynamin 2 (left) and cortactin (right). Boxed areas correspond to enlarged images shown below. Bar, $10 \mu \mathrm{m}$ (top and bottom panels), $2.8 \mu \mathrm{m}$ (middle panels). (C) Representative images acquired by immunoelectron microscopy showing the localization of dynamin 2 (top three panels) and cortactin (bottom three panels) in filopodia of serum-stimulated H1299 cells. Immunoreactive dynamin 2 and cortactin were present along F-actin bundles (arrowheads). Scale bar, 20 nm. (D) Immunoprecipitation (IP) results demonstrating an in vivo interaction between dynamin 2 and cortactin. H1299 cells were co-transfected with GFP-tagged dynamin 2 (Dyn2-GFP) and either myc-tagged wild-type cortactin (Cort WT-myc, left) or cortactin $\Delta \mathrm{SH} 3$ (Cort $\Delta \mathrm{SH} 3$-myc, right). The protein complexes were immunoprecipitated using a polyclonal anti-myc antibody or preimmune $\operatorname{IgG}(\mathrm{IgG})$, and then visualized by western blotting (WB) with monoclonal anti-GFP or anti-myc antibodies. Total cell lysates $(4,10$ and $20 \mu \mathrm{g})$ were also analyzed (input).

and wild-type cortactin resulted in the formation of long and thick F-actin bundles (Fig. 5A, + Dyn2 + Cort WT), which were similar to those formed by dynamin 1 and cortactin (Fig. 5A, + Dyn1 + Cort WT). On the other hand, the long and thick F-actin bundles were much less evident in the presence of dynamin 2 and cortactin W525K or $\Delta$ SH3 (Fig. 5A, + Dyn2 + Cort W525K or + Dyn $2+$ Cort $\Delta \mathrm{SH} 3$ ).

To localize dynamin 2 and cortactin to F-actin bundles, the preformed F-actin bundles were used for immunofluorescent staining. Dynamin 2 and cortactin colocalized as bright dots along F-actin bundles (Fig. 5B, left). The localization of dynamin 2 and cortactin was similar to that of the dynamin 1-cortactin complex (Fig. 5B, right) (12).

Lastly, we examined whether actin bundling by the dynamin 2-cortactin complex can affect F-actin stability.
To address this, the depolymerization kinetics of preformed pyrene-labeled F-actin were examined after the solution was diluted 10 -fold with buffer. In the presence of dynamin 2 and cortactin, the rate of depolymerization by dilution decreased to a level comparable to that induced by $\alpha$-actinin, an actincrosslinking protein, indicating that dynamin 2 and cortactin stabilize F-actin bundles (Fig. 5C). These results indicate that the dynamin 2-cortactin complex stabilizes F-actin bundles in filopodia prior to cell migration.

\section{Discussion}

The involvement of dynamin in the dynamics of cancer cells such as cell migration, invasion, and metastasis has been reported (18). However, the precise role of dynamin in these 

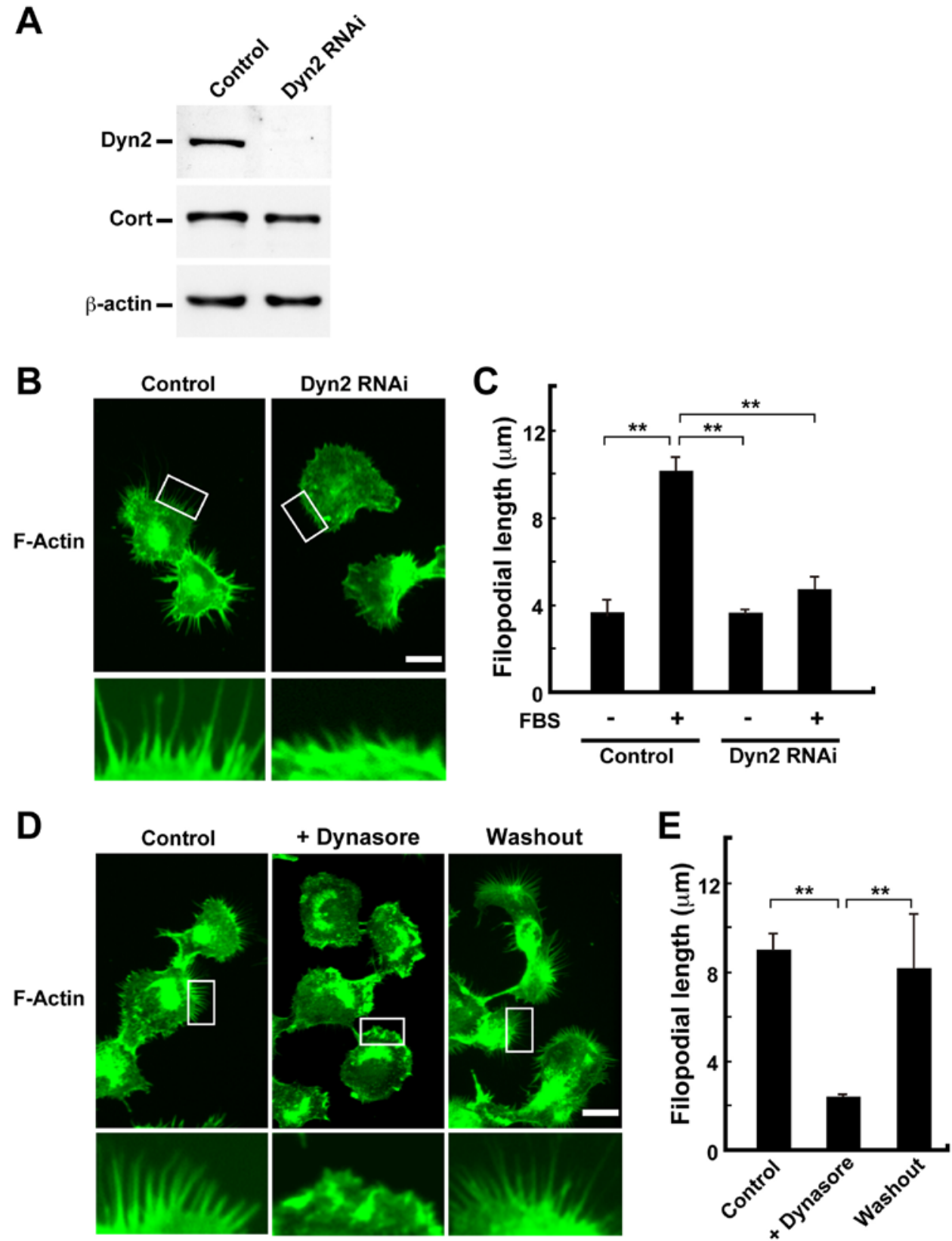

Figure 3. Knockdown of dynamin 2 decreases filopodial formation in H1299 cells. (A) Western blotting showing knockdown of dynamin 2 (Dyn2) expression by RNAi in H1299 cells. $\beta$-actin served as the control. Three micrograms of cell lysate from each sample was analyzed by gel electrophoresis. (B) F-actin was visualized in H1299 cells by Alexa Fluor 488-phalloidin staining after knockdown of dynamin 2. Extensive filopodial formation was observed in cells after serum stimulation (left). Filopodial formation was inhibited in dynamin 2-silenced cells (right). Boxed areas correspond to enlarged images shown below. Scale bar, $20 \mu \mathrm{m}$ (upper panels), $5 \mu \mathrm{m}$ (lower panels). (C) Filopodial length in H1299 cells cultured in the presence or absence of serum. The cells were visualized by fluorescent confocal microscopy, and filopodial length was measured as described in Materials and methods. (D) Inhibition of filopodial formation by dynasore in serum-stimulated H1299 cells. Serum-starved cells were incubated with $240 \mu$ M dynasore for 30 min, and then stimulated with $10 \%$ FBS for $45 \mathrm{~min}$ in the presence of $240 \mu \mathrm{M}$ dynasore (middle). Thereafter, dynasore was removed, and the cells were incubated in serum-containing medium for 45 min (right). For the negative control, cells were cultured in the presence of $3 \%$ DMSO (left). All steps were performed at $37^{\circ} \mathrm{C}$. (E) Analysis of filopodial formation in the H1299 cells shown in (D). The cells were analyzed by fluorescent confocal microscopy, and filopodial length was measured. Results in (C) and (E) represent the means \pm SEM from three independent experiments.

cellular processes is not entirely clear. We recently reported that actin bundling by the dynamin 1-cortactin complex is crucial for neurite extension in developing neurons (12). In this study, we examined the possibility that a similar F-actinbundling mechanism is involved in the migration of H1299 cells, a human non-small cell lung carcinoma cell line.

We showed that cortactin and dynamin 2 mostly colocalized along F-actin bundles in filopodia of serum-stimulated
H1299 cells (Fig. 2). Pharmacological inhibition of dynamin 2 by Dynasore, Dynole 34-2 or MitMAB decreased cell migration (Fig. 1) and filopodial formation (Fig. 3). Furthermore, filopodia were shorter in dynamin 2- and cortactin-depleted cells than in control cells (Figs. 3 and 4). In cortactin-silenced cells, the exogenous expression of wild-type cortactin rescued the punctate-like localization of dynamin 2 and filopodial formation (Fig. 4). Both dynamin 2 and cortactin bundled 
A
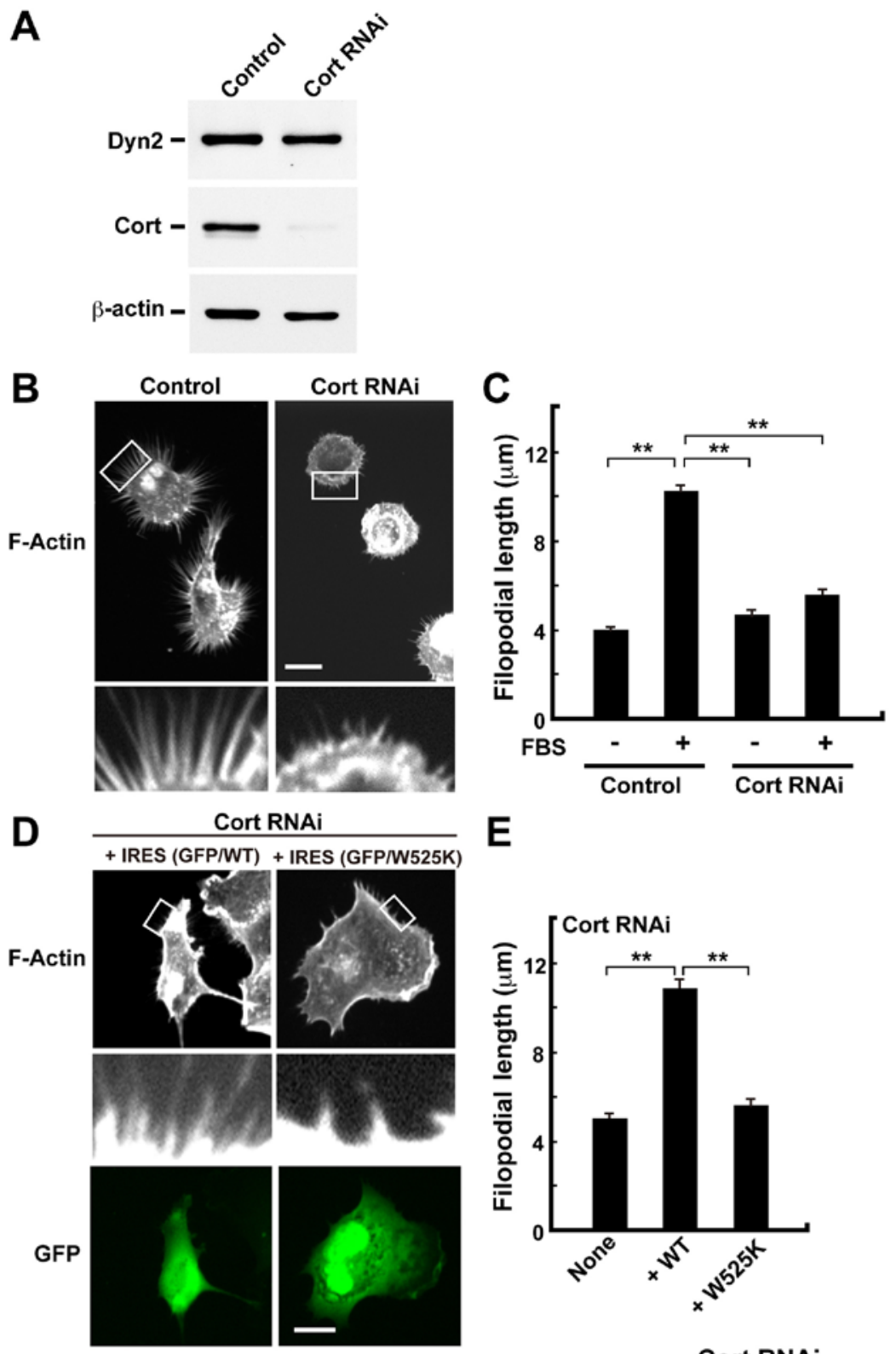

Cort RNAi

E

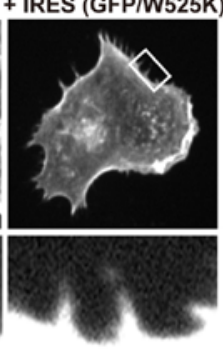

$\mathbf{F}$
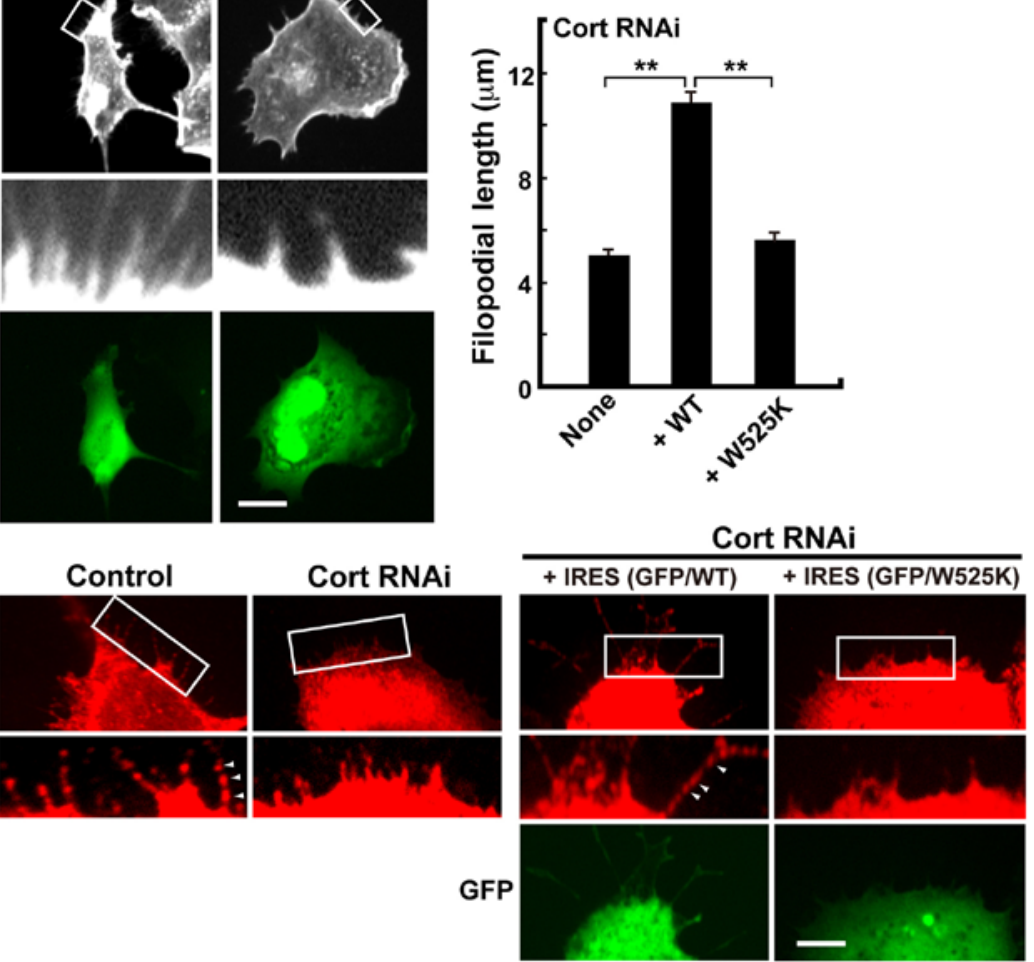

Figure 4. Knockdown of cortactin decreases filopodial formation in H1299 cells. (A) Western blotting showing knockdown of cortactin expression by RNAi in H1299 cells. $\beta$-actin was used as the control. Three micrograms of cell lysate from each sample was analyzed by gel electrophoresis. (B) F-actin was visualized in serum-stimulated H1299 cells by Alexa Fluor 488-phalloidin staining. Boxed areas correspond to enlarged images shown below. Similar to results from dynamin 2-depleted cells, filopodial formation decreased in cortactin-depleted cells (right). Scale bar, $20 \mu \mathrm{m}$ (upper panels), $5 \mu \mathrm{m}$ (lower panels). (C) Analysis of filopodial formation in H1299 cells cultured with or without serum. The samples were analyzed by fluorescent confocal microscopy, and the filopodial length was measured. (D) Expression of wild-type cortactin rescues filopodial formation. Cortactin-depleted H1299 cells were transfected with rat wild-type cortactin (left) or cortactin W525K (right) cloned into the pIRES2-AcGFP1 expression vector. Boxed areas correspond to enlarged images shown (middle panels). Transfected cells were identified by GFP expression (bottom panels). Scale bar, $20 \mu \mathrm{m}$ (top and bottom panels), $3.5 \mu \mathrm{m}$ (middle panels). (E) Analysis of filopodial formation in $\mathrm{H} 1299$ cells. The samples were analyzed by fluorescent confocal microscopy, and the filopodial length was measured. Results in (C) and (E) represent the means \pm SEM from three independent experiments. (F) Rescue of the punctate-like localization of dynamin 2 along F-actin bundles in filopodia by re-expression of cortactin in cortactin-depleted cells. Cortactin-depleted H1299 cells (left panels) were transfected with rat wild-type cortactin or cortactin W525K cloned into the pIRES2-AcGFP1 expression vector (right panels). The cells were immunostained with an anti-dynamin 2 antibodies. Boxed areas correspond to enlarged images shown below. Transfected cells were identified by GFP expression (right bottom panels). Scale bar, $5 \mu \mathrm{m}$ (top and right bottom panels), $2.3 \mu \mathrm{m}$ (left bottom and right middle panels). 
A
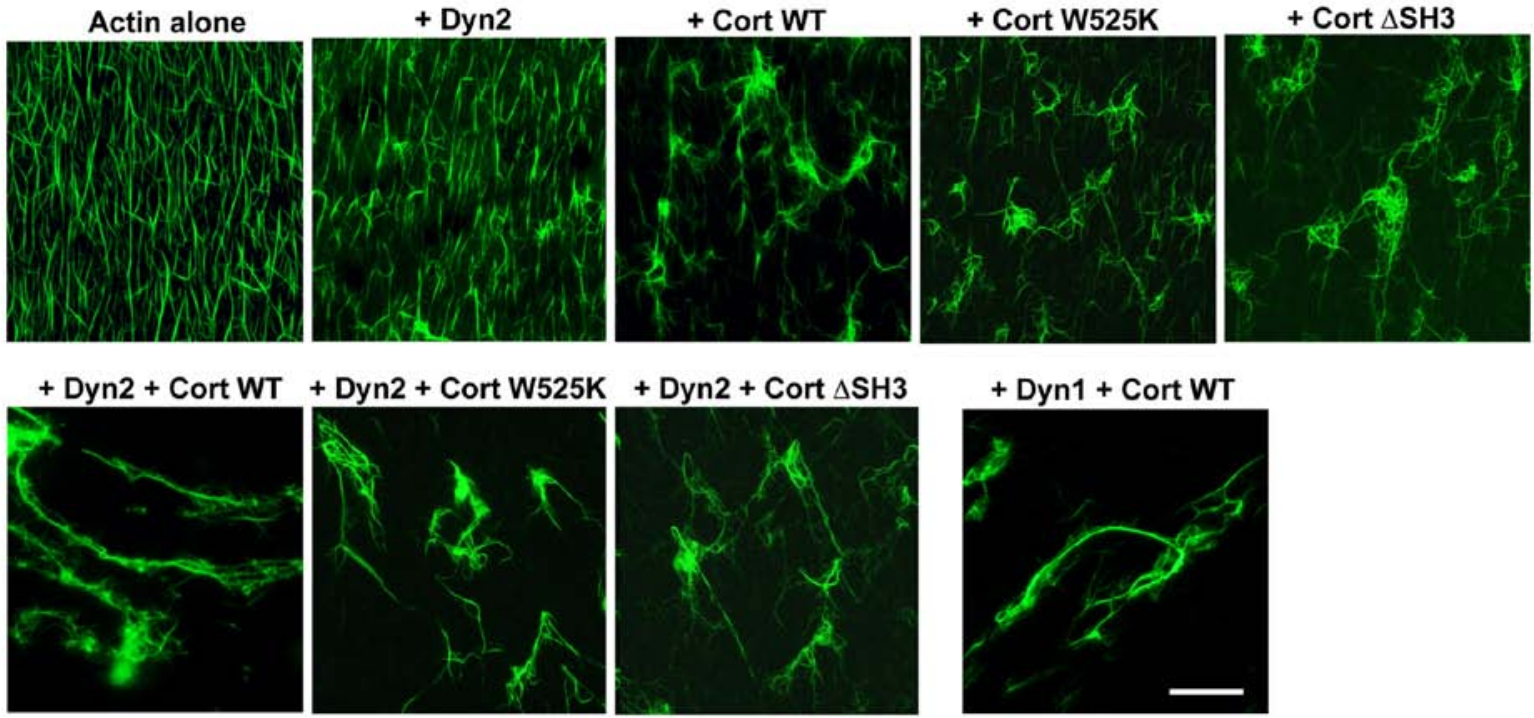

B
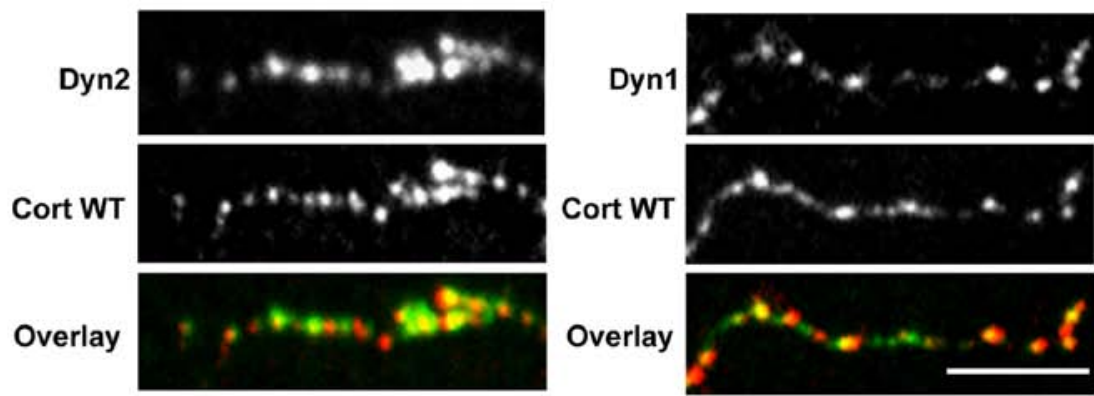

C

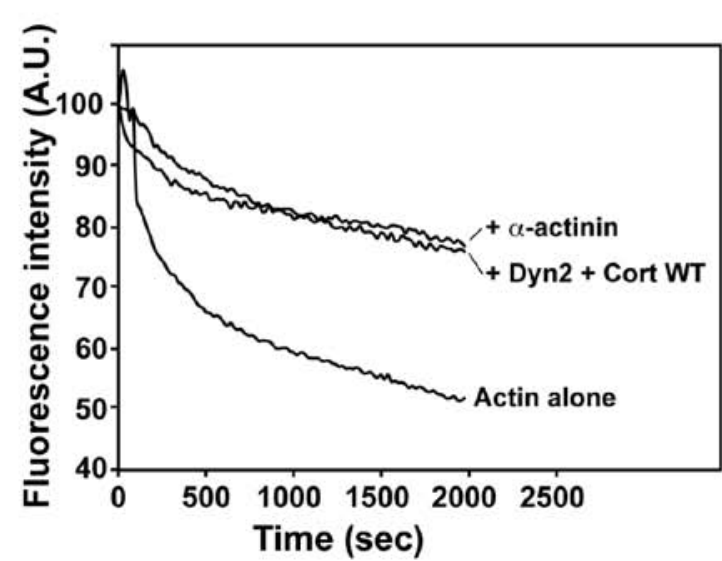

Figure 5. Actin bundling by dynamin 2 and cortactin stabilizes F-actin bundles. (A) Long F-actin bundles were formed in the presence of dynamin 2 and cortactin (lower left). Preformed F-actin $(3.3 \mu \mathrm{M})$ was incubated with or without the indicated proteins $(5 \mu \mathrm{M}$ each). F-actin was visualized with Alexa Fluor 488-phalloidin. Scale bar, $30 \mu \mathrm{m}$. (B) Representative images acquired by fluorescent microscopy showing the localization of dynamin and cortactin along F-actin bundles. Actin bundles were formed in vitro by incubating dynamin 2 with wild-type cortactin (left) or dynamin 1 and wild-type cortactin (right). Protein colocalization was performed by double-immunofluorescence. Scale bar, $2 \mu \mathrm{m}$. (C) Kinetics of F-actin disassembly induced in 10-fold diluted preformed pyrene-labeled F-actin solution with buffer. F-actin bundles disassembled in the presence of dynamin 2 and cortactin, as well as in the presence of $5 \mu \mathrm{M} \alpha$-actinin. The rate of F-actin bundle disassembly was measured by pyrene-fluorescence.

F-actin, and these proteins increased F-actin stability (Fig. 5). These results indicate that dynamin 2 and cortactin participate in cancer cell migration by stabilizing F-actin bundles in filopodia.

Dynamin assembles at the neck of deeply invaginated endocytic pits (30). Upon GTP hydrolysis, however, dynamin undergoes a conformational change, resulting in the fission of endocytic pits and release of endocytic vesicles (31-33). In addition, dynamin 1 forms a ring-like complex with cortactin, which switches from an open to a closed state upon GTP hydrolysis. This change promotes the bundling of F-actin filaments (12). The mechanism of actin bundling mediated by the dynamin 2-cortactin complex is similar to that of the dynamin 1-cortactin complex, because dynamin 2 and cortactin also facilitated the formation of long and thick F-actin bundles to which they colocalized (Fig. 5). This mechanochemical 


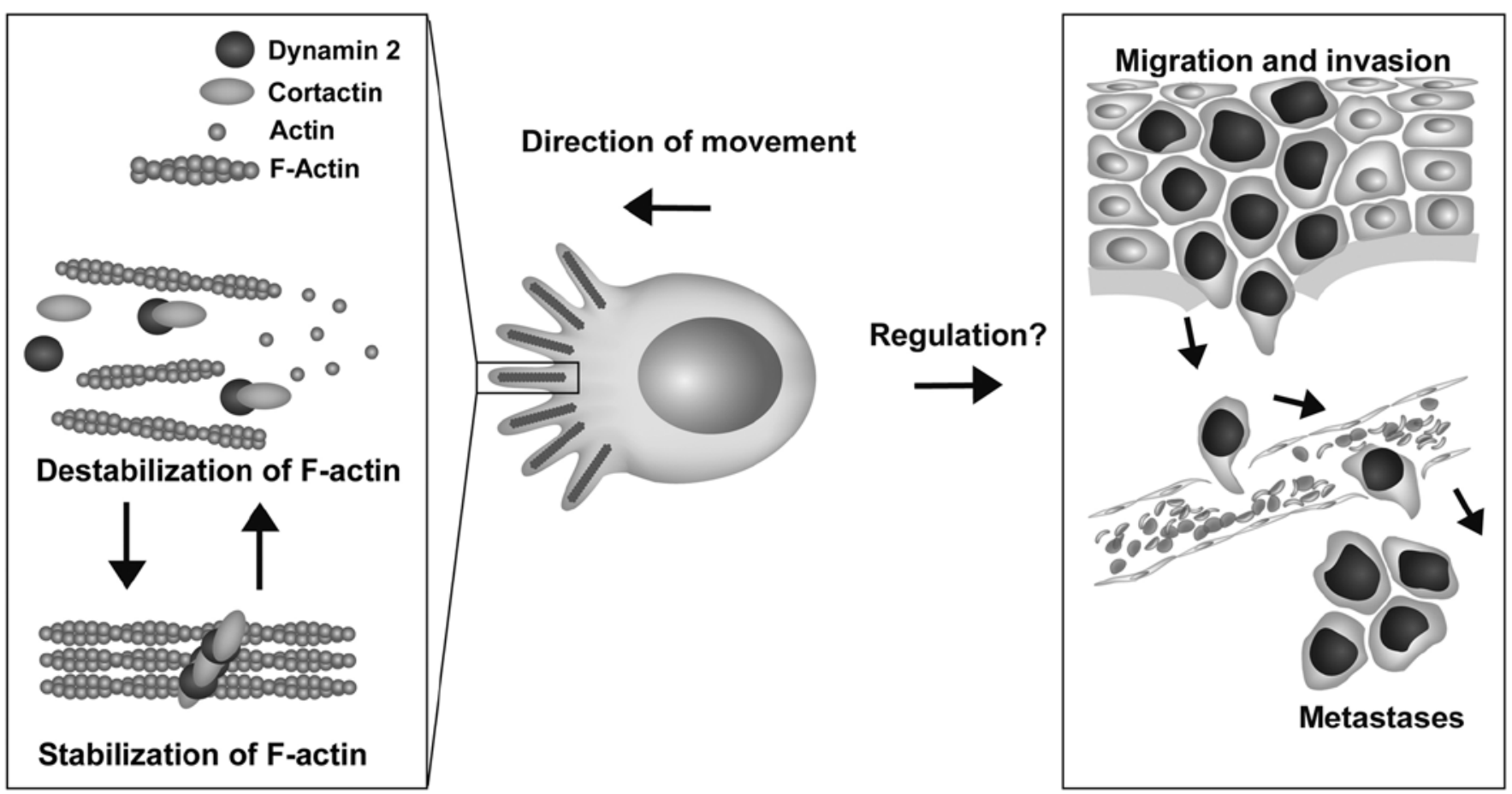

Figure 6. Putative role of the dynamin 2-cortactin complex in cancer cell migration. The dynamin 2-cortactin complex bundles actin filaments, which stabilize filopodia. In addition, the dynamin 2-cortactin complex participates in the formation of pseudopodia. The regulation of actin by dynamin 2 and cortactin may also be involved in cancer cell invasion and metastasis.

property may be critical for the formation of F-actin bundles in filopodia of other cell types as well (Fig. 6). Additional studies are needed to determine the precise mechanism.

Dynamin associates with tumorigenesis, particularly tumor cell migration and invasion. For example, increased dynamin 2 expression potentiates the migration and invasion of pancreatic ductal cancer cells (25), and tyrosine phosphorylated dynamin 2 promotes the growth and invasiveness of glioblastomas (34). Thus, the involvement of dynamin in the formation of F-actin bundles might promote cancer malignancy.

In conclusion, we showed that dynamin 2 and cortactin participate in the formation of F-actin bundles, which stabilize filopodia in migrating cancer cells. Taken together, these results suggest that dynamin might be a potential molecular target for anticancer therapy.

\section{Acknowledgements}

The authors thank Yuki Masuoka, Dr Shun-AI Li, and Nana Okazaki for technical assistance. This study was supported in part by grants from the Ministry of Education, Science, Sports, and Culture of Japan (grant no. 26670201 to H.Y.; grant no. 15K1533007 to K.T.), the Astellas Foundation for Research on Metabolic Disorders (to H.Y.), and the Japan Foundation for Applied Enzymology (to H.Y.).

\section{References}

1. Arjonen A, Kaukonen R and Ivaska J: Filopodia and adhesion in cancer cell motility. Cell Adhes Migr 5: 421-430, 2011.

2. Ridley AJ: Life at the leading edge. Cell 145: 1012-1022, 2011.

3. Takei K, Slepnev VI, Haucke V and De Camilli P: Functional partnership between amphiphysin and dynamin in clathrinmediated endocytosis. Nat Cell Biol 1: 33-39, 1999.
4. Mettlen M, Pucadyil T, Ramachandran R and Schmid SL: Dissecting dynamin's role in clathrin-mediated endocytosis. Biochem Soc Trans 37: 1022-1026, 2009.

5. Praefcke GJ and McMahon HT: The dynamin superfamily: Universal membrane tubulation and fission molecules? Nat Rev Mol Cell Biol 5: 133-147, 2004.

6. Cao H, Garcia F and McNiven MA: Differential distribution of dynamin isoforms in mammalian cells. Mol Biol Cell 9: 2595-2609, 1998

7. McNiven MA, Kim L, Krueger EW, Orth JD, Cao $\mathrm{H}$ and Wong TW: Regulated interactions between dynamin and the actin-binding protein cortactin modulate cell shape. J Cell Biol 151: 187-198, 2000.

8. Baldassarre M, Pompeo A, Beznoussenko G, Castaldi C, Cortellino S, McNiven MA, Luini A and Buccione R: Dynamin participates in focal extracellular matrix degradation by invasive cells. Mol Biol Cell 14: 1074-1084, 2003.

9. Ochoa GC, Slepnev VI, Neff L, Ringstad N, Takei K, Daniell L, Kim W, Cao H, McNiven M, Baron R, et al: A functional link between dynamin and the actin cytoskeleton at podosomes. J Cell Biol 150: 377-389, 2000.

10. Torre E, McNiven MA and Urrutia R: Dynamin 1 antisense oligonucleotide treatment prevents neurite formation in cultured hippocampal neurons. J Biol Chem 269: 32411-32417, 1994.

11. Kurklinsky S, Chen J and McNiven MA: Growth cone morphology and spreading are regulated by a dynamin-cortactin complex at point contacts in hippocampal neurons. J Neurochem 117: 48-60, 2011.

12. Yamada H, Abe T, Satoh A, Okazaki N, Tago S, Kobayashi K, Yoshida Y, Oda Y, Watanabe M, Tomizawa K, et al: Stabilization of actin bundles by a dynamin 1/cortactin ring complex is necessary for growth cone filopodia. J Neurosci 33: 4514-4526, 2013.

13. Gold ES, Underhill DM, Morrissette NS, Guo J, McNiven MA and Aderem A: Dynamin 2 is required for phagocytosis in macrophages. J Exp Med 190: 1849-1856, 1999.

14. Otsuka A, Abe T, Watanabe M, Yagisawa $\mathrm{H}$, Takei $\mathrm{K}$ and Yamada H: Dynamin 2 is required for actin assembly in phagocytosis in Sertoli cells. Biochem Biophys Res Commun 378: 478-482, 2009.

15. Faelber K, Posor Y, Gao S, Held M, Roske Y, Schulze D, Haucke V, Noé F and Daumke O: Crystal structure of nucleotidefree dynamin. Nature 477: 556-560, 2011. 
16. Ford MG, Jenni S and Nunnari J: The crystal structure of dynamin. Nature 477: 561-566, 2011.

17. Wu H, Reynolds AB, Kanner SB, Vines RR and Parsons JT: Identification and characterization of a novel cytoskeletonassociated pp60src substrate. Mol Cell Biol 11: 5113-5124, 1991

18. MacGrath SM and Koleske AJ: Cortactin in cell migration and cancer at a glance. J Cell Sci 125: 1621-1626, 2012.

19. Ammer AG and Weed SA: Cortactin branches out: Roles in regulating protrusive actin dynamics. Cell Motil Cytoskeleton 65: 687-707, 2008.

20. Yamada H, Abe T, Li SA, Masuoka Y, Isoda M, Watanabe M, Nasu Y, Kumon H, Asai A and Takei K: Dynasore, a dynamin inhibitor, suppresses lamellipodia formation and cancer cell invasion by destabilizing actin filaments. Biochem Biophys Res Commun 390: 1142-1148, 2009.

21. Yamada H, Abe T, Li SA, Tago S, Huang P, Watanabe M, Ikeda S, Ogo N, Asai A and Takei K: N'-[4-(dipropylamino)benzylidene]2-hydroxybenzohydrazide is a dynamin GTPase inhibitor that suppresses cancer cell migration and invasion by inhibiting actin polymerization. Biochem Biophys Res Commun 443: 511-517, 2014.

22. Mooren OL, Kotova TI, Moore AJ and Schafer DA: Dynamin2 GTPase and cortactin remodel actin filaments. J Biol Chem 284: 23995-24005, 2009.

23. Masaike Y, Takagi T, Hirota M, Yamada J, Ishihara S, Yung TM, Inoue T, Sawa C, Sagara H, Sakamoto S, et al: Identification of dynamin-2-mediated endocytosis as a new target of osteoporosis drugs, bisphosphonates. Mol Pharmacol 77: 262-269, 2010

24. Slepnev VI, Ochoa GC, Butler MH and De Camilli P: Tandem arrangement of the clathrin and AP-2 binding domains in amphiphysin 1 and disruption of clathrin coat function by amphiphysin fragments comprising these sites. J Biol Chem 275: 17583-17589, 2000 .

25. Eppinga RD, Krueger EW, Weller SG, Zhang L, Cao H and McNiven MA: Increased expression of the large GTPase dynamin 2 potentiates metastatic migration and invasion of pancreatic ductal carcinoma. Oncogene 31: 1228-1241, 2012.
26. Macia E, Ehrlich M, Massol R, Boucrot E, Brunner C and Kirchhausen T: Dynasore, a cell-permeable inhibitor of dynamin. Dev Cell 10: 839-850, 2006.

27. Hill TA, Gordon CP, McGeachie AB, Venn-Brown B, Odell LR, Chau N, Quan A, Mariana A, Sakoff JA, Chircop M, et al: Inhibition of dynamin mediated endocytosis by the dynoles-synthesis and functional activity of a family of indoles. J Med Chem 52: 3762-3773, 2009.

28. Quan A, McGeachie AB, Keating DJ, van Dam EM, Rusak J, Chau N, Malladi CS, Chen C, McCluskey A, Cousin MA, et al: Myristyl trimethyl ammonium bromide and octadecyl trimethyl ammonium bromide are surface-active small molecule dynamin inhibitors that block endocytosis mediated by dynamin I or dynamin II. Mol Pharmacol 72: 1425-1439, 2007.

29. Schafer DA, Weed SA, Binns D, Karginov AV, Parsons JT and Cooper JA: Dynamin 2 and cortactin regulate actin assembly and filament organization. Curr Biol 12: 1852-1857, 2002.

30. Takei K, McPherson PS, Schmid SL and De Camilli P: Tubular membrane invaginations coated by dynamin rings are induced by GTP-gamma S in nerve terminals. Nature 374: 186-190, 1995.

31. Sweitzer SM and Hinshaw JE: Dynamin undergoes a GTP-dependent conformational change causing vesiculation. Cell 93: 1021-1029, 1998

32. Takei K, Haucke V, Slepnev V, Farsad K, Salazar M, Chen H and De Camilli P: Generation of coated intermediates of clathrinmediated endocytosis on protein-free liposomes. Cell 94: 131-141, 1998.

33. Roux A, Uyhazi K, Frost A and De Camilli P: GTP-dependent twisting of dynamin implicates constriction and tension in membrane fission. Nature 441: 528-531, 2006.

34. Feng H, Liu KW, Guo P, Zhang P, Cheng T, McNiven MA, Johnson GR, Hu B and Cheng SY: Dynamin 2 mediates PDGFR $\alpha$-SHP-2-promoted glioblastoma growth and invasion. Oncogene 31: 2691-2702, 2012. 